\title{
2
}

Finish line $Q$

\section{The finish line}

Hi again!

We will repeat the same procedure as during the kick-off session. Please make sure that you are wearing the heart rate monitor (Zephyr BioHarness)

Again, we will start by asking you some questions about the course of the measurement week.

Activity at work

Think of work as the things that you have to do such as paid or unpaid work, study/training, and household chores or gardening.

Vigorous-intensity activities are activities that require hard physical effort and cause large increases in breathing or heart rate.

Moderate-intensity activities are activities that require moderate physical effort and cause small increases in breathing or heart rate

Does your work involve vigorous-intensity activities for at least 10 minutes continuously? more info

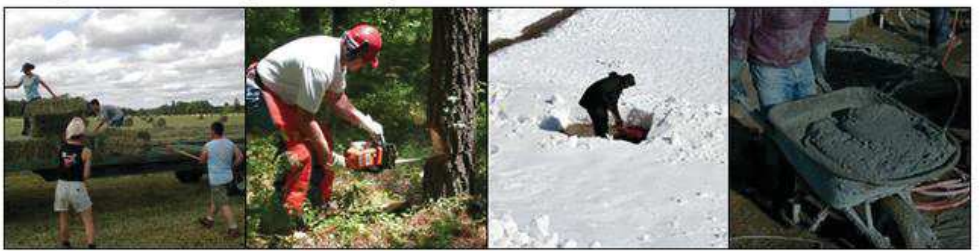

- Yes

- No

In the last 7 days, on how many days did you do vigorous-intensity activities as part of your work?

Typically, how much time do you spend doing vigorous-intensity activities at work on such a day? $1: 00$

Please enter the duration as hours:minutes, separated by ":" (e.g., 2:30)

Does your work involve moderate-intensity activity for at least 10 minutes continuously? more info

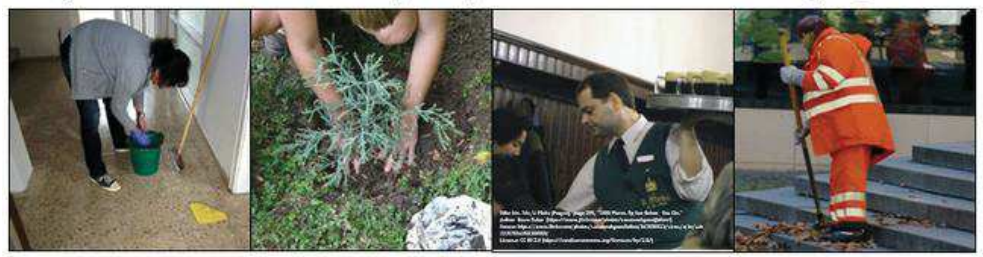

(e) Yes

- No 
Travel to and from places

The following questions exclude the physical activities at work that you have already mentioned.

Now think about the usual way in which you travel to and from places. Do not include walking or cycling for leisure or sports.

In the last 7 days, on how many days did you use each of the following methods of travel to get to and from places?

\begin{tabular}{|c|c|c|c|c|}
\hline & Did not use it & on 1.3 days per week & on $4-5$ days per week & on $6-7$ days per week \\
\hline Walk & 0 & 0 & • & 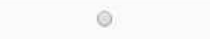 \\
\hline Bicycle & - & $\odot$ & $\odot$ & $\ominus$ \\
\hline Electric bicycle & - & e & ○ & 0 \\
\hline Motorcycle or moped & - & $\circ$ & ○ & $\odot$ \\
\hline Public transport & 0 & - & 0 & 0 \\
\hline Car or van & ○ & - & ○ & ○ \\
\hline
\end{tabular}

In the last 7 days, did you walk or use a bicycle for at least 10 minutes continuously to get to and from places?

- Walk

Bicycle

- Electric bicycle

No

In the last 7 days, on how many days did you walk for at least 10 minutes continuously to get to and from places? 5

Typically, how much time do you spend walking on such a day?

$0: 40$

Please enter the duration as hours:minutes, separated by ":" (e.g., 2:30) 


\section{Q iिPASTR}

Recreational activities

For the next questions exclude the work and transport activities that you have already mentioned. Now think about sports, fitness and recreational activities (leisure), including going for a walk or on a cycle tour.

Vigorous-intensity activities are activities that require hard physical effort and cause large increases in breathing or heart rate

Moderate-intensity activities are activities that require moderate physical effort and cause small increases in breathing or heart rate.

Do you do any vigorous-intensity sports, fitness or recreational (leisure) activities for at least 10 minutes continuously? more info

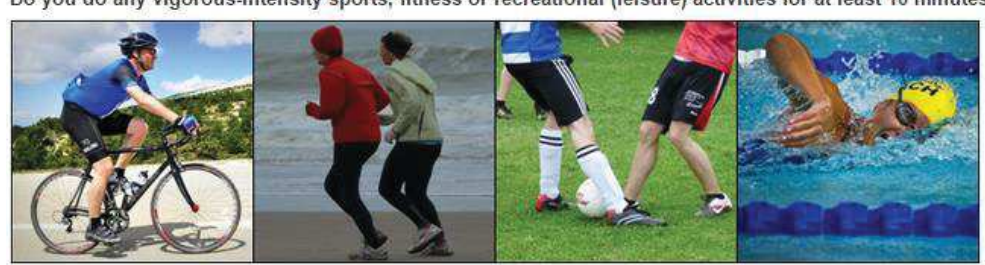

Yes

- No

Do you do any moderate-intensity sports, fitness or recreational (leisure) activities for at least 10 minutes continuously? more info

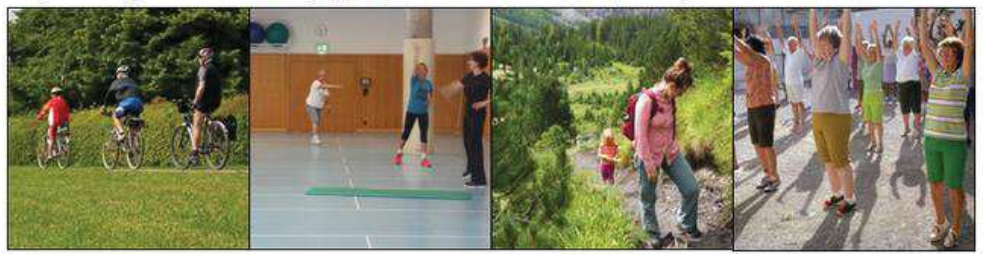

(.) Yes

No

In the last 7 days, on how many days did you do moderate-intensity sports, fitness or recreational (leisure) activities? 1

Typically, how much time do you spend doing moderate-intensity sports, fitness or recreational activities on such a day? 1:30

Please enter the duration as hours minutes, separated by ":" (e.g., 2:30).

\section{Previous}

The finish line

Sedentary behaviour

The following question is about sitting or reclining at work, at home, getting to and from places, or with friends. Time spent sleeping should not be included.

For example: time spent sitting at a desk; eating; travelling in car, bus or train; reading; watching television; or using the computer

In the last 7 days, how much time did you spend sitting or reclining on a typical day?

$8: 30$

Please enter the duration as hours.minutes, separated by ":" (e.g., 2:30) 